\title{
AN ANALYTICAL STUDY OF THE EARLY STAGES OF UNSTEADY FREE CONVECTIVE FLOW FROM A DIFFERENTIALLY HEATED ROTATING SPHERE AT LARGE GRASHOF NUMBERS
}

\author{
S.J.D. D'ALESSIO \\ Faculty of Mathematics, University of Waterloo, Canada.
}

\begin{abstract}
This research investigates the unsteady free convective flow of a viscous incompressible fluid from a differentially heated rotating sphere. The flow is assumed to remain laminar and to possess equatorial and azimuthal symmetry. The governing Navier-Stokes and energy equations are posed in terms of a scaled stream function - vorticity formulation and are solved subject to no-slip and specified surface temperature conditions. At $\mathrm{t}=0$ an impulsive heat flux is applied in the form of a jump in surface temperature. An asymptotic solution valid for large Grashof numbers and small times following the impulsive startup is constructed. Two small parameters have been identified and based on this the flow variables are expanded in a double series in powers of these parameters. The non-zero leading-order terms in the asymptotic expansions have been determined analytically and the corresponding heat transfer coefficient has been found. Future work will involve obtaining numerical solutions.

Keywords: asymptotic, free convection, incompressible, rotation, thin flow, viscous.
\end{abstract}

\section{INTRODUCTION}

Free convective flow from a differentially heated rotating sphere occurs naturally in the atmosphere and thus represents an important and well-studied problem. Numerous investigations, some of which are listed in [1], focussing on various aspects have been devoted to this subject. The present study represents a continuation of the work reported by D'Alessio et al. [1] which emphasized the stability of the steady-state flow for small Rayleigh numbers.

The current work addresses unsteady free convective flow at large Grashof numbers. Here, the flow is taken to be laminar and to possess equatorial and azimuthal symmetry while the fluid is viscous, incompressible and Newtonian. As shown in Figure 1 the sphere rotates about the polar axis and with respect to a reference frame that rotates with the sphere the fluid is initially isothermal and at rest. At $t=0$ the fluid is impulsively set into motion as a result of a specified surface temperature distribution that varies sinusoidally with the polar angle and exceeds the temperature of the surrounding ambient fluid. An asymptotic solution describing the abrupt startup has been derived.

The paper is structured as follows. In the next section the mathematical formulation of the problem is presented. The governing equations are introduced in dimensionless form along with their corresponding boundary and initial conditions. Following that, in section 3, the equations are rescaled to better capture and resolve the early development of the flow. Then in section 4 an asymptotic solution procedure valid for small times and large Grashoh numbers is outlined. The paper is summarized in section 5 .

\section{MATHEMATICAL FORMULATION}

Because of the assumed azimuthal and equatorial symmetry, the governing unsteady Navier-Stokes and energy equations can be expressed in terms of a stream function $(\psi)$ and 


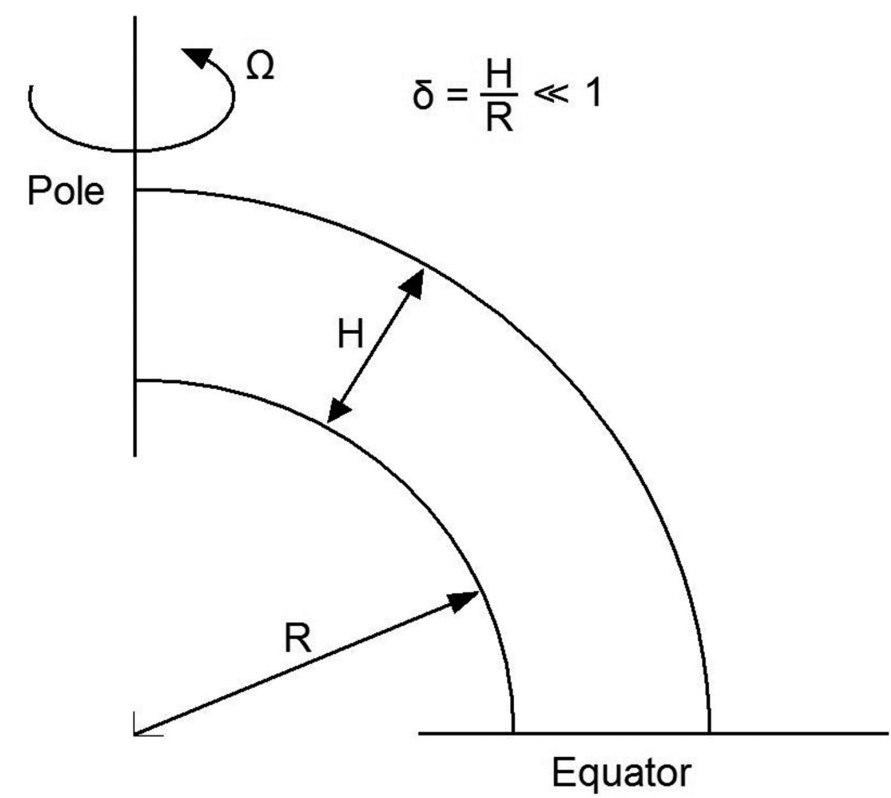

Figure 1: The flow set up.

vorticity $(\omega)$. The velocity components $v_{r}, v_{\theta}$ in the $r, \theta$ directions, respectively, are related to the stream function through the relations

$$
v_{r}=\frac{1}{r^{2} \sin \theta} \frac{\partial \psi}{\partial \theta}, v_{\theta}=-\frac{1}{r \sin \theta} \frac{\partial \psi}{\partial r}
$$

In the spherical coordinates $(r, \theta)$ and cast in dimensionless form the equations can be formulated as [1,2]

$$
\begin{gathered}
\omega=-D^{2} \psi \\
\frac{\partial \omega}{\partial t}+\frac{1}{r^{2} \sin \theta} \frac{\partial(\psi, \omega)}{\partial(\theta, r)}+\sin \theta \frac{\partial T}{\partial \theta}+\frac{2 \omega}{r^{2} \sin ^{2} \theta}\left(\cos \theta \frac{\partial \psi}{\partial r}-\frac{\sin \theta}{r} \frac{\partial \psi}{\partial \theta}\right) \\
-\left(\frac{2 W}{r^{2} \sin ^{2} \theta}+\frac{2}{F r}\right)\left(\cos \theta \frac{\partial W}{\partial r}-\frac{\sin \theta}{r} \frac{\partial W}{\partial \theta}\right)=\frac{1}{\sqrt{G r}} D^{2} \omega \\
\frac{1}{\sqrt{G r}} D^{2} W-\frac{\partial W}{\partial t}=\frac{1}{r^{2} \sin \theta} \frac{\partial(\psi, W)}{\partial(\theta, r)}-\frac{2}{F r}\left(\cos \theta \frac{\partial \psi}{\partial r}-\frac{\sin \theta}{r} \frac{\partial \psi}{\partial \theta}\right) \\
\frac{\partial T}{\partial t}+\frac{1}{r^{2} \sin \theta} \frac{\partial(\psi, T)}{\partial(\theta, r)}=\frac{1}{\sqrt{G r} P r} \nabla^{2} T
\end{gathered}
$$


In the above $t$ denotes time, $r$ is the radial coordinate, and $\theta$ is the angle with the polar axis. The flow variable $W$ denotes the scaled zonal velocity while $T$ is the scaled temperature. The dimensionless parameters appearing in the above equations include the Grashof number $(\mathrm{Gr})$, the Froude number $(\mathrm{Fr})$ and the Prandtl number $(\mathrm{Pr})$ which are defined as follows

$$
G r=\frac{\alpha g R^{3} \Delta T}{v^{3}}, F r=\frac{U}{\Omega R}, \operatorname{Pr}=\frac{v}{\kappa} .
$$

Here, the fluid properties $v, \kappa$ and $\alpha$ represent the kinematic viscosity, thermal diffusivity and thermal expansion coefficient, respectively, whereas $g$ is the acceleration due to gravity, $R$ is the radius of the sphere, $\Delta T$ is the maximum temperature difference between the surface and the top of the fluid layer, $\Omega$ is the rotation rate about the polar axis and $U=\sqrt{\alpha g R \Delta T}$ is the convective velocity scale which is chosen to reflect the convective nature of the problem for early times. The thinness of the fluid layer will be taken into account later when we introduce the boundary-layer coordinate $y$.

In deriving the vorticity transport equation (2) we have made the Boussinesq approximation whereby the fluid density $(\rho)$ varies linearly with temperature according to

$$
\rho=\rho_{a}\left[1-\alpha\left(T-T_{a}\right)\right]
$$

where $\rho_{a}$ represents the reference density corresponding to the ambient temperature $T_{a}$. The time and length are scaled as follows

$$
\tilde{t} \rightarrow \frac{R}{U} t, \tilde{r} \rightarrow R r
$$

whereas the adopted scaling for the flow variables is given by

$$
(\tilde{\psi}, \tilde{\omega}, \tilde{W}, \tilde{T}) \rightarrow\left(R^{2} U \psi, U \omega, R U W, T_{a}+(\Delta T) T\right)
$$

In the above, the tilde denotes a dimensional quantity. Lastly, the differential operators $D^{2}, \nabla^{2}$ and $\partial(A, B) / \partial(x, y)$ are defined as follows

$$
\begin{aligned}
& D^{2}=\frac{\partial^{2}}{\partial r^{2}}+\frac{1}{r^{2}} \frac{\partial^{2}}{\partial \theta^{2}}-\frac{\cot \theta}{r^{2}} \frac{\partial}{\partial \theta}, \\
& \nabla^{2}=\frac{\partial^{2}}{\partial r^{2}}+\frac{2}{r} \frac{\partial}{\partial r}+\frac{1}{r^{2}} \frac{\partial^{2}}{\partial \theta^{2}}+\frac{\cot \theta}{r^{2}} \frac{\partial}{\partial \theta}, \\
& \frac{\partial(A, B)}{\partial(x, y)}=\frac{\partial A}{\partial x} \frac{\partial B}{\partial y}-\frac{\partial A}{\partial y} \frac{\partial B}{\partial x} .
\end{aligned}
$$

Equations (1)-(4) are to be solved in the region 


$$
0 \leq \theta \leq \frac{\pi}{2}, 1 \leq r \leq 1+\delta,
$$

subject to the no-slip boundary conditions given by

$$
\psi=\frac{\partial \psi}{\partial r}=W=0 \text { on } r=1 \text { and } r=1+\delta
$$

The dimensionless parameter $\delta=H / R$ represents the thickness of the fluid layer with $H$ denoting the dimensional thickness. As we will see, the parameter does not enter the problem because for early times the flow will be confined to a very thin thermal boundary layer adjacent to the surface of the sphere. Effectively, we will replace the no-slip conditions at $r=1+$ $\delta$ by the far-field conditions $\psi, \omega, W, T \rightarrow 0$ at a sufficiently large distance from the surface. This condition corresponds to a quiescent far-field flow maintained at a constant temperature. Even though the fluid layer is thin, initially everything is happening near the surface and it will take some time before the flow reaches the outer boundary located at $r=1+\delta$.

The assumed symmetry requires that we impose the following conditions at $\theta=0, \pi / 2$

$$
\psi=\omega=W=0 \text { along } \theta=0 \text { and } \psi=\omega=\frac{\partial W}{\partial \theta}=0 \text { along } \theta=\frac{\pi}{2} .
$$

We observe that the stream function is overspecified while the vorticity is underspecified. Later we will explain how the extra conditions for the stream function can be used to furnish the missing conditions for the vorticity. For two-dimensional isothermal flows Dennis and Quartapelle [3] have shown that the vorticity field satisfies integral constraints which can be derived from the no-slip boundary conditions using Green's second identity. D'Alessio and Perera [4] have extended the use of integral constraints to two-dimensional free convective flows.

At $t=0$ an impulsive heat flux is applied in the form of a jump in surface temperature. Prior to $t=0$ both the surface of the sphere and the surrounding fluid are maintained at a temperature $T=0$. Then, at $t=0$ the surface temperature is suddenly set to

$$
T=1-\gamma \cos ^{2} \theta \text { on } r=1
$$

to model the equator-to-pole decrease in surface temperature. Here, the parameter $\gamma$ represents the ratio of the maximum difference in surface temperature to the maximum difference in temperature between the surface and the top of the fluid layer. The dimensionless temperature has been scaled so that the maximum difference in temperature between the surface and the top of the fluid layer is unity, and the constant temperature at the top of the fluid layer is zero. The purpose of the impulsive startup is to keep the problem as general as possible, provided that we can properly handle the initial temperature discontinuity.

Since the fluid initially has a uniform temperature and the motion starts from rest, the initial conditions are simply

$$
\psi(r, \theta, t)=\omega(r, \theta, t)=W(r, \theta, t)=T(r>1, \theta, t)=0 \text { at } t=0 .
$$

Lastly, at $\theta=0$ and $\theta=\pi / 2$ we apply the zero heat-flux condition 


$$
\frac{\partial T}{\partial \theta}=0 \text { along } \theta=0 \text { and } \theta=\frac{\pi}{2} .
$$

\section{RESCALED EQUATIONS}

To better resolve the early stages of the flow and heat transfer process following the impulsive startup at $t=0$, we introduce the boundary-layer coordinate, $y$, which is related to $\mathrm{r}$ through the relationship

$$
r=e^{\lambda y} \text { where } \lambda=\sqrt{\frac{4 t}{\sqrt{G r}}} .
$$

Essentially, this change of variable stretches the thin thermal-boundary layer with $\lambda$ describing the diffusive growth of the evolving boundary layer. Another advantage of working in terms of the coordinate $y$ is that the physical coordinate $r$ becomes a moving coordinate; that is, lines of constant $y$ expand in time when plotted in a Cartesian coordinate system. This is ideal from a numerical point of view since the grid lines are alive and allowed to expand with the growing boundary layer to ensure adequate resolution during the early stages.

The parameter $\lambda$ appearing in the transformation given by (5) follows from the physics of the problem. To see this, we examine the dominant terms in the temperature equation immediately following the impulsive startup. Since the flow starts from rest and gradients in the $r$ direction are expected to be much larger than those in the $\theta$ direction, equation (4) can be simplified by retaining only the key terms which are

$$
\frac{\partial T}{\partial t}=\frac{1}{\sqrt{G r} P r}\left(\frac{\partial^{2} T}{\partial r^{2}}+\frac{2}{r} \frac{\partial T}{\partial r}\right) .
$$

The above heat conduction equation can be written more compactly as

$$
\frac{\partial \chi}{\partial t}=\frac{1}{\sqrt{G r} P r} \frac{\partial^{2} \chi}{\partial r^{2}},
$$

where $\chi=r T$. The solution satisfying $\chi=1-\gamma \cos ^{2} \theta$ at $r=1$ and $\chi \rightarrow 0$ as $r \rightarrow \infty 1$ is given by

$$
\chi(r, \theta)=\left(1-\gamma \cos ^{2} \theta\right) \operatorname{erfc}\left(\sqrt{p r} \sqrt{\frac{\sqrt{G r}}{4 t}} r\right),
$$

Where $\operatorname{erfc}(x)=1-\operatorname{erf}(x)$ is the complimentary error function while

$$
\operatorname{erf}(x)=\frac{2}{\sqrt{\pi}} \int_{0}^{x} e^{-u^{2}} d u
$$

is the error function. Expressed this way we see that the solution naturally involves the parameter $\lambda$.

In terms of the coordinate y equations (1)-(4) get transformed to 


$$
\begin{aligned}
& \frac{\partial^{2} \psi}{\partial y^{2}}-\lambda \frac{\partial \psi}{\partial y}+\lambda^{2} \frac{\partial^{2} \psi}{\partial \theta^{2}}-\lambda^{2} \cot \theta \frac{\partial \psi}{\partial \theta}=-\lambda^{2} e^{2 \lambda y} \omega \\
& \frac{\partial^{2} \omega}{\partial y^{2}}+2 y e^{2 \lambda y} \frac{\partial \omega}{\partial y}=4 t e^{2 \lambda y}\left(\frac{\partial \omega}{\partial t}+\sin \theta \frac{\partial T}{\partial \theta}\right)+\frac{4 t e^{-\lambda y}}{\lambda \sin \theta}\left(\frac{\partial \psi}{\partial \theta} \frac{\partial \omega}{\partial y}-\frac{\partial \psi}{\partial y} \frac{\partial \omega}{\partial \theta}\right) \\
& -\frac{8 t}{\lambda}\left(\frac{e^{-\lambda y} W}{\sin ^{2} \theta}+\frac{e^{\lambda y}}{F r}\right)\left(\cos \theta \frac{\partial w}{\partial y}-\lambda \sin \theta \frac{\partial W}{\partial \theta}\right) \\
& +\lambda\left(\frac{\partial \omega}{\partial y}+\lambda \cos \theta \frac{\partial \omega}{\partial \theta}-\lambda \frac{\partial^{2} \omega}{\partial \theta^{2}}\right)+\frac{8 t e^{-\lambda y} \omega}{\lambda \sin ^{2} \theta}\left(\cos \theta \frac{\partial \psi}{\partial y}-\lambda \sin \theta \frac{\partial \psi}{\partial \theta}\right), \\
& \frac{\partial^{2} W}{\partial y^{2}}+2 y e^{2 \lambda y} \frac{\partial W}{\partial y}=4 t e^{2 \lambda y} \frac{\partial W}{\partial t}+\lambda\left(\frac{\partial W}{\partial y}-\lambda \frac{\partial^{2} W}{\partial \theta^{2}}+\lambda \cos \theta \frac{\partial w}{\partial \theta}\right) \\
& +\frac{4 t e^{-\lambda y}}{\lambda \sin \theta}\left(\frac{\partial \psi}{\partial \theta} \frac{\partial W}{\partial y}-\frac{\partial \psi}{\partial y} \frac{\partial W}{\partial \theta}\right)-\frac{8 t e^{\lambda y}}{\lambda F r}\left(\cos \theta \frac{\partial \psi}{\partial y}-\lambda \sin \theta \frac{\partial \psi}{\partial \theta}\right) \\
& \frac{1}{\operatorname{Pr}} \frac{\partial^{2} T}{\partial y^{2}}+2 y e^{2 \lambda y} \frac{\partial T}{\partial y}=4 t e^{2 \lambda y} \frac{\partial T}{\partial t} \\
& -\frac{\lambda}{\operatorname{Pr}}\left(\frac{\partial T}{\partial y}+\lambda \frac{\partial^{2} T}{\partial \theta^{2}}+\lambda \cot \theta \frac{\partial T}{\partial \theta}\right)+\frac{4 t e^{-\lambda y}}{\lambda \sin \theta}\left(\frac{\partial \psi}{\partial \theta} \frac{\partial T}{\partial y}-\frac{\partial \psi}{\partial y} \frac{\partial T}{\partial \theta}\right),
\end{aligned}
$$

while the associated boundary and initial conditions become

$$
\begin{gathered}
\psi=\frac{\partial \psi}{\partial y}=W=0 \text { and } T=1-\gamma \cos ^{2} \theta \text { on } y=0, \\
\psi, \omega, W, T \rightarrow 0 \text { as } y \rightarrow \infty, \\
\psi(y, \theta, t)=\omega(y, \theta, t)=W(y, \theta, t)=T(y>0, \theta, t)=0 \text { at } t=0 .
\end{gathered}
$$

As a final note we emphasize that although the boundary-layer coordinate y is utilized, the fully nonlinear Navier-Stokes and energy equations are to be solved and not the simplified thermal boundary-layer equations. We next outline how to construct an asymptotic solution to the system of equations (6)-(9) for small $\mathrm{t}$ and large $G r$.

\section{ASYMPTOTIC SOLUTION}

Here we present a multiple series expansion technique to derive an asymptotic solution for the early development of the flow and heat transfer process. The key advantage offered by this method is that it provides a systematic procedure which reduces the problem to a set of linear ordinary differential equations.

The procedure involves expanding the flow variables in powers of the parameter $\lambda$ which will be small if $t$ is small or if $G r$ is large. It turns out that if this is done the resulting equations are still too complicated to solve analytically. If $t$ is taken to be small and $G r$ is taken to 
be large then we can identify two small parameters appearing in the problem, $\lambda$ and $t$, and it is then possible to expand the flow variables in a double series as follows. First, we expand $T$, $\omega, \psi$ and $W$ in a series of the form

$$
\begin{aligned}
& T=T_{0}+\lambda T_{1}+\lambda^{2} T_{2}+\cdots, \\
& \omega=\omega_{0}+\lambda \omega_{1}+\lambda^{2} \omega_{2}+\cdots, \\
& \psi=\psi_{0}+\lambda \psi_{1}+\lambda^{2} \psi_{2}+\cdots, \\
& W=W_{0}+\lambda W_{1}+\lambda^{2} W_{2}+\cdots
\end{aligned}
$$

Then each $T_{n}, \omega_{n}, \psi_{n}, W_{n}(\mathrm{n}=0,1,2, \ldots)$ are further expanded in a series of the form

$$
\begin{aligned}
& T_{n}(y, \theta, t)=T_{n 0}(y, \theta)+t T_{n 1}(y, \theta)+t^{2} T_{n 2}(y, \theta)+\cdots, \\
& \omega_{n}(y, \theta, t)=\omega_{n 0}(y, \theta)+t \omega_{n 1}(y, \theta)+t^{2} \omega_{n 2}(y, \theta)+\cdots, \\
& \psi_{n}(y, \theta, t)=\psi_{n 0}(y, \theta)+t \psi_{n 1}(y, \theta)+t^{2} \psi_{n 2}(y, \theta)+\cdots, \\
& W_{n}(y, \theta, t)=W_{n 0}(y, \theta)+t W_{n 1}(y, \theta)+t^{2} W_{n 2}(y, \theta)+\cdots .
\end{aligned}
$$

We note that when performing a double expansion the internal orders of magnitudes between the expansion parameters should be taken into account. In our case, $\lambda$ and $t$ will be the equal when $t=4 / \sqrt{G r}$. Thus, for a fixed value of $G r$ this procedure is expected to be valid for times that are of order $1 / \sqrt{G r}$ provided that $G r$ is sufficiently large. Fortunately, asymptotic expansions are known to have the redeeming feature that they often provide good results outside the domain of validity. Since the governing equations also involve powers of the exponential function $e^{\lambda y}$, the expansion $e^{\lambda y}=1+\lambda y+\lambda^{2} y^{2} / 2+\ldots$ will also have to be used. We point out that this double expansion procedure has been successfully applied to many two-dimensional problems involving unsteady flow and heat transfer from cylindrical bodies, such as in $[4,5]$, to list a few.

Substituting the above series into equations (6)-(9) produces a hierarchy of problems at various orders. At each order the solution strategy is similar; that is, we first solve for the temperature and then use the generated solution to determine the vorticity. Next, we solve for the stream function and then finally we determine the zonal velocity.

The leading-order term for the temperature, $T_{0}$, satisfies the equation

$$
\frac{1}{\operatorname{Pr}} \frac{\partial^{2} T_{0}}{\partial y^{2}}+2 y \frac{\partial T_{0}}{\partial y}=4 t \frac{\partial T_{0}}{\partial t} .
$$

It can be shown that the solution satisfying the conditions (10) and (11) is

$$
T_{0}(y, \theta)=\left(1-\gamma \cos ^{2} \theta\right) \operatorname{erfc}(\sqrt{\operatorname{Pr}} y)
$$

which bears a close resemblance to the solution for $\chi(\mathrm{r}, \theta)$ obtained earlier in the previous section. 
We next solve for the leading-order term in the vorticity expansion. Owing to conditions (11) and (12) it is an easy exercise to show that $\omega_{0}=0$ and that the leading non-zero term is

The homogeneous solution, $\omega_{01, \mathrm{~h}}$, is given by [6]

$$
\frac{\partial^{2} \omega_{01}}{\partial y^{2}}+2 y \frac{\partial \omega_{01}}{\partial y}-4 \omega_{01}=4 \sin \theta \frac{\partial T_{0}}{\partial \theta} .
$$

$$
\omega_{01, h}(y, \theta)=A(\theta)\left(y^{2}+\frac{1}{2}\right)+B(\theta)\left[y e^{-y^{2}}+\sqrt{\pi}\left(y^{2}+\frac{1}{2}\right) \operatorname{erf}(y)\right]
$$

where $A(\theta), B(\theta)$ are functions which are yet to be determined. By variation of parameters the particular solution, $\omega_{01, \mathrm{p}}$, has been found to be

$$
\omega_{01, p}(y, \theta)=\gamma \sin \theta \sin 2 \theta\left(y^{2} \operatorname{erfc}(y)+y^{2}+\frac{1}{2} \operatorname{erf}(y)-\frac{y}{\sqrt{\pi}} e^{-y^{2}}\right),
$$

for $\operatorname{Pr}=1$ and

$$
\begin{gathered}
\omega_{01, p}(y, \theta)=4 \gamma \sin \theta \sin 2 \theta\left(\frac{y^{2}}{2} \operatorname{erfc}(\sqrt{\operatorname{Pr} y})+\frac{\left(y^{2}+\frac{1}{2}\right)}{2(1-\operatorname{Pr})} \operatorname{erf}(\sqrt{\operatorname{Pr} y})\right. \\
\left.+\frac{\sqrt{\operatorname{Pr} y}}{2 \sqrt{\pi(1-P r)}} e^{-P r y^{2}}\right)
\end{gathered}
$$

for $\operatorname{Pr} \neq 1$. In order to satisfy the far-field condition $\omega_{01} \rightarrow 0$ as $y \rightarrow \infty$ we must have that $A(\theta)=-\sqrt{ } \pi B(\theta)-\gamma \sin \theta \sin 2 \theta$ for $\operatorname{Pr}=1$ while $\mathrm{A}(\theta)=-\sqrt{\pi B}(\theta)$ for $\operatorname{Pr} \neq 1$. Thus, the general solution becomes

$$
\begin{aligned}
& \omega_{01}(y, \theta)=B(\theta)\left[y e^{-y^{2}}-\sqrt{\pi}\left(y^{2}+\frac{1}{2}\right) \operatorname{erfc}(\mathrm{y})\right] \\
& -\gamma \sin \theta \sin 2 \theta\left(y^{2}+\frac{1}{2}\right)+\omega_{01, p}(y, \theta),
\end{aligned}
$$

for $\operatorname{Pr}=1$ while

$$
\omega_{01}(y, \theta)=B(\theta)\left[y e^{-y^{2}}-\sqrt{\pi}\left(y^{2}+\frac{1}{2}\right) \operatorname{erfc}(y)\right]+\omega_{01, p}(y, \theta)
$$

for $\operatorname{Pr} \neq 1$. To determine the remaining unknown function $\mathrm{B}(\theta)$ we enforce the integral constraint

$$
\int_{0}^{\pi / 2} \int_{0}^{\infty} \omega_{01}(y, \theta) \sin \theta d y d \theta=0
$$

It can be shown that this condition follows directly from Green's second identity [3]. After some algebra the solution for $\omega_{01}$ for $\operatorname{Pr}=1$ becomes 


$$
\omega_{01}(y, \theta)=\gamma \sin \theta \sin 2 \theta\left[\left(3 y^{2}+\frac{1}{2}\right) \operatorname{erfc}(y)-\frac{3 y}{\sqrt{\pi}} e^{-y^{2}}\right],
$$

whereas for $\operatorname{Pr} \neq 1$ we obtain

$$
\begin{aligned}
& \omega_{01}(y, \theta)=2 \gamma \sin \theta \sin 2 \theta\left[\frac{\left(y^{2}+\frac{1}{2}\right)}{\sqrt{\operatorname{Pr}}(1-\operatorname{Pr})}[\operatorname{erfc}(y)-\sqrt{\operatorname{Pr}} \operatorname{erfc}(\sqrt{\operatorname{Pr}} y)]\right. \\
& \left.+y^{2} \operatorname{erfc}(\sqrt{\operatorname{Pr}} y)+\frac{y}{\sqrt{\pi \operatorname{Pr}}(1-\operatorname{Pr})}\left(\operatorname{Pr} e^{-\operatorname{Pry}^{2}}-e^{-y^{2}}\right)\right] .
\end{aligned}
$$

Next, we solve for the stream function. From a straight-forward calculation we find that the leading non-zero term is $\psi_{21}$ and satisfies

$$
\frac{\partial^{2} \psi_{21}}{\partial y^{2}}=-\omega_{01}
$$

Integrating twice and applying the no-slip and impermeability conditions

$$
\psi_{21}=\frac{\partial \psi_{21}}{\partial y}=0 \text { at } y=0
$$

yields

$$
\psi_{21}(y, \theta)=-\gamma \sin \theta \sin 2 \theta\left[\frac{y^{2}}{4}\left(y^{2}+1\right) \operatorname{erfc}(y)+\frac{1}{16} \operatorname{erf}(y)-\frac{y}{8 \sqrt{\pi}}\left(1+2 y^{2}\right) e^{-y^{2}}\right],
$$

for $\operatorname{Pr}=1$ while

$$
\begin{aligned}
& \psi_{21}(y, \theta)=-\frac{2 \gamma \sin \theta \sin 2 \theta}{(1-\operatorname{Pr})}\left[\frac{y^{2}}{12 \sqrt{\operatorname{Pr}}}\left(y^{2}+3\right) \operatorname{erfc}(y)-\frac{y^{2}}{12}\left(\operatorname{Pry} y^{2}+3\right) \operatorname{erfc}(\sqrt{\operatorname{Pr}} y)\right. \\
& \left.+\frac{1}{16 \operatorname{Pr}}[\operatorname{erf}(\sqrt{\operatorname{Pr}} y)-\sqrt{\operatorname{Pr}} \operatorname{erf}(y)]+\frac{y}{24 \sqrt{\pi} \operatorname{Pr}}\left[\left(5+2 \operatorname{Pr}^{2}\right) e^{-P r y^{2}}-\left(5+2 y^{2}\right) e^{-y^{2}}\right]\right]
\end{aligned}
$$

for $\operatorname{Pr} \neq 1$. We note that $\psi_{21}$ does not satisfy the far-field condition. Since the stream function is overspecified we chose to apply the surface conditions instead of the far-field condition when solving for $\psi_{21}$.

Lastly, we solve for the zonal velocity. It follows that the leading non-zero term is $W_{12}$ and satisfies

$$
\frac{\partial^{2} W_{12}}{\partial y^{2}}+2 y \frac{\partial W_{12}}{\partial y}-10 W_{12}=-\frac{8}{F r} \cos \theta \frac{\partial \psi_{21}}{\partial y}
$$


subject to the conditions $W_{12}=0$ at $y=0$ and $W_{12} \rightarrow 0$ as $\mathrm{y} \rightarrow \infty$. Although it may be possible to find a closed form analytical solution to this equation, it will likely be very complicated and will involve Parabolic Cylinder Functions [6].

Summarizing, we have identified the following leading-order non-zero terms in the expansions; the initial solution is well approximated by

$$
\begin{aligned}
& T(y, \theta, t)=T_{0}(y, \theta)+O(\lambda), \\
& \omega(y, \theta, t)=t \omega_{01}(y, \theta)+O\left(t^{2}+\lambda\right), \\
& \psi(y, \theta, t)=\lambda^{2} t \psi_{21}(y, \theta)+O\left(\lambda^{2} t^{2}+\lambda^{3}\right), \\
& W(y, \theta, t)=\lambda t^{2} W_{12}(y, \theta)+O\left(\lambda t^{3}+\lambda^{2}\right),
\end{aligned}
$$

with the understanding that $W_{12}$ may need to be determined numerically.

An important quantity related to the flow is the heat transfer coefficient. For a sphere the dimensionless average heat transfer coefficient is defined as

$$
N u=-\frac{1}{2 \pi} \int_{0}^{2 \pi} \int_{0}^{\pi}\left(\frac{\partial T}{\partial r}\right)_{r=1} \sin \theta d \theta d \phi
$$

In the case of azimuthual symmetry the above simplifies to

$$
N u=-\int_{0}^{\pi}\left(\frac{\partial T}{\partial r}\right)_{r=1} \sin \theta d \theta
$$

For small $t$ and large $\mathrm{Gr}$ we can derive an expression for $\mathrm{Nu}$ using the constructed approximate solution for $T$ given by $T_{0}(y, \theta)$. Using $r=\mathrm{e}^{\lambda \mathrm{y}}$ and $\lambda=2 \sqrt{\mathrm{t}} / \sqrt{\mathrm{Gr}}$ it follows that

$$
N u \approx 2\left(1-\frac{\gamma}{3}\right) \sqrt{\frac{\operatorname{Pr} \sqrt{G r}}{\pi t}} .
$$

We note that if $\gamma=3$, then $N u=0$ as a result of the prescribed surface temperature profile. Also, as $t \rightarrow 0, N u \rightarrow \infty$ as a result of the initial temperature discontinuity.

\section{CONCLUSIONS}

Free convective flow from a differentially heated rotating sphere was discussed in this paper. An approximate analytical solution was found in the form of an asymptotic expansion which is valid for large Grashof numbers and small times following the impulsive startup. Based on the analytical solution, the heat transfer coefficient was then determined.

Future work will involve obtaining numerical solutions to this problem which can provide solutions for much larger times. The asymptotic solution constructed in this paper can play an important role in the numerical solution procedure because, in essence, it can be used to furnish initial conditions for the flow variables at a small time beyond the initial singularity when the solutions become well behaved. For large $G r$ it may not be possible for the numerical solution procedure to cope with the discontinuity in temperature at $t=0$. At the very least the approximate solution can be used to accelerate the numerical solution procedure. For 
smaller values of $G r$ the numerical solution procedure may be able to start at $t=0$. Then, the approximate analytical solution can be compared with the numerically generated solution and can therefore be used to test the numerical solution procedure. For large $G r$ and sufficiently large times the hope is that the numerical solutions will generate flow patterns that are consistent with those observed in the atmosphere.

\section{ACKNOWLEDGEMENTS}

Financial support for this research was provided by the Faculty of Mathematics at the University of Waterloo.

\section{REFERENCES}

[1] D’Alessio, S.J.D., Leung, N. \& Wan, J.W.L., Stability of differentially heated flow from a rotating sphere. Journal of Computational and Applied Mathematics, 291, pp. 209-224, 2016.

https://doi.org/10.1016/j.cam.2015.03.025

[2] Goldstein, S. (Ed.). Modern Developments in Fluid Dynamics, Clarendon Press, Oxford, 1938.

[3] Dennis, S.C.R. \& Quartapelle, L., Some uses of Green's theorem in solving the Navier-Stokes equations. International Journal Of Numerical Methods in Fluids, 9 , pp. 871-890, 1989.

https://doi.org/10.1002/fld.1650090802

[4] D'Alessio, S.J.D. \& Perera, R.N., Unsteady free convection from elliptic cylinders at large Grashof numbers. International Journal of Heat Mass Transfer, 52, pp. 59405953, 2009.

https://doi.org/10.1016/j.ijheatmasstransfer.2009.08.008

[5] Badr, H.M. \& Dennis, S.C.R., Time dependent viscous flow past an impulsively started rotating and translating circular cylinder. Journal of Fluid Mechanics, 158, pp. 447488, 1985.

https://doi.org/10.1017/s0022112085002725

[6] Bateman, H. \& Erdelyi, A., Higher Transcendental Functions, Volume 2, McGraw-Hill, 1953. 